\title{
Estrés laboral y expresión de ira en trabajadores de una unidad minera en tiempos de covid - 19, Juliaca
}

\section{Work stress and expression of anger in workers of a mining unit in times of covid - 19, Juliaca}

\author{
Luiggi Zamora Deza'
}

\begin{abstract}
RESUMEN
Objetivo: determinar si existe relación significativa entre el estrés laboral y expresión de ira en trabajadores de una unidad minera en tiempos de covid - 19, Juliaca 2021. Metodología: diseño no experimental, de corte transversal, tipo correlación y enfoque cuantitativo. La población estuvo constituida por 60 trabajadores, la muestra fue no probabilística por conveniencia, las características de la población muestraron que la mayoría pertenece al género masculino, sus edades oscilan entre 20 a 50 años y todos ellos están registrados en la unidad minera. Los instrumentos utilizados fueron el cuestionario de estrés laboral OIT - OMS, este instrumento alcanza un índice de alta confiabilidad con el coeficiente alpha de Cronbach de 0,961, y un grado de validez mínimo $\mathrm{V}=0,98$; y el inventario de expresión de ira Estado - Rasgo (STAXI 2) alcanza un índice de alta confiabilidad con el coeficiente de alpha de Cronbach de 0,943 y un grado de validez adecuado $V=1$. Resultados: existe una relación directa y significativa ( $r h o=, 577 ; p<0.05)$ entre las variables de estudio. Conclusión: a mayor nivel de estrés laboral, mayor será el nivel de expresión de ira.
\end{abstract}

Palabras clave: estrés laboral; expresión de ira.

\section{ABSTRACT}

Objective: to determine if there is a significant relationship between work stress and expression of anger in workers of a mining unit in times of covid-19, Juliaca 2021. Methodology: non-experimental design, cross-sectional, correlation type and quantitative approach. The population consisted of 60 workers, the sample was non-probabilistic for convenience, the characteristics of the population showed that the majority belong to the male gender, their ages range from 20 to 50 years and all of them are registered in the mining unit. The instruments used were the ILO - WHO work stress questionnaire. This instrument achieves a high reliability index with Cronbach's alpha coefficient of 0.961, and a minimum degree of validity $V=0.98$; and the State-Trait anger expression inventory (STAXI 2) reaches a high reliability index with Cronbach's alpha coefficient of 0.943 and an adequate degree of validity $V=1$. Results: there is a direct and significant relationship (rho $=.577 ; p<0.05$ ) between the study variables. Conclusion: the higher the level of work stress, the higher the level of expression of anger.

Keywords: work stress; expression of anger. 


\section{INTRODUCCIÓN}

Se cree que el $50 \%$ de los trabajadores en los países industrializados (EE.UU., China, Alemania y Reino Unido) consideran su trabajo "mentalmente demandante", mientras que el 20 y $50 \%$ de los trabajadores en los países en desarrollo (Costa Rica - Honduras) pueden tener exposiciones riesgosas, teniendo el conocimiento del trabajo ya que cada año existen aproximadamente 120 millones de lesiones por los accidentes de trabajo, 200.000 fatalidades ocupacionales, y entre 68 y 157 millones de casos de enfermedades ocupacionales en la fuerza laboral global (Houtman, 2015).

En el sector de servicio, la reducción del personal, la paralización, limitación salarial, el incremento de la carga de trabajo y de las metas de rendimiento, la prolongación de las jornadas de trabajo, y el desarrollo al trabajo momentáneo y la sub - contratación son factores de tensión que afecta e incita al estrés laboral y a la violencia en el lugar de trabajo, existencia de efectos negativos en el rendimiento y la eficiencia de las organizaciones, incrementando los casos de licencia por enfermedad, ausentismo y el sistema de rotación en los trabajadores de acuerdo a la Organización Mundial del Trabajo (OIT, 2003). Cada empresa optó nuevas maneras de trabajar y convivir en sociedad debido a la pandemia del covid 19, lo cual, cada trabajador tuvo que cambiar rotundamente su estilo de vida personal, familiar y manejo de actividades en la empresa (Becerra Hernandez, 2020).

Según el barómetro de la compañía francesa Edenred Perú 2021, esta pandemia ha generado un impacto psicológico negativo permitiendo secuelas emocionales como la confusión, ira, aburrimiento, sentimiento de soledad, etc., de 1056 personas encuestadas, entre varones y mujeres, el $54.7 \%$ indicó que realiza teletrabajo parcial o total; de este grupo el $7.3 \%$ indica que su obligación laboral incrementó entre 1 a 5 hrs al día, obteniendo un desbalance entre la vida personal y labora Tello (2021).

El Covid - 19 llegó a Perú en momentos vulnerables en el mercado de trabajo, pasando por un alto aumento económico y creación de oficios; entre el mes de abril - junio del 2020, la población con empleo disminuyó en 6.7 millones de personas y sumando la desocupación y el crecimiento del empleo informal; se notó en la capital del Perú - Lima Metropolitana, la marcada desocupación en varones jóvenes entre 14 a 24 años, de igual forma con personas de nivel educativo bajo, merece la pena subrayar que el nivel de ingreso laboral real, sea visto como hace nueve años atrás. Posteriormente se vió al año 2020 con una caída de 1.5 millones de personas con empleo a comparación del año 2019 (Gamero \& Pérez, 2020).

El trabajo remoto como consecuencia de la pandemia es uno de los excesos de responsabilidades, problemas en casa, la baja autoestima, realizando horas extras, disminución del sueño, situaciones inesperadas, entre otros; prueba de ello se tiene a Sara Mendoza Figueroa, gerente de Marketing de Visma Latinoamérica (Expertos en Soluciones para Recursos Humanos) indica que en un estudio de Korn Ferry (Mendoza, 2020) 2.000 profesionales consultados, el $75 \%$ afirma que el estrés laboral perjudica sus relaciones y su vida familiar, siendo las principales preocupaciones: el impacto financiero de la crisis $49 \%$, perder a un ser querido $40 \%$, enfermarse $37 \%$, incertidumbre acerca de cómo el virus impactará a su familia y relaciones $25 \%$, y el estrés laboral o trabajo excesivo a raíz de esta situación $23 \%$ (Diario Correo, 2021).

De acuerdo con la Organización Mundial de la Salud (OMS) en México con el $75 \%$ se encuentran las personas más estresadas del mundo, por encima de China que tiene el $73 \%$ y Estados Unidos con el $59 \%$. El estrés laboral producido por el desequilibrio de las exigencias y presiones a los que un trabajador se enfrenta Expok (2017).

El Estrés es uno de los factores más comunes y frecuentes en el mundo laboral, siendo un riesgo para la salud de todos los colaboradores, causando muchas enfermedades tanto físicas como psíquicas. La globalización económica avanza a pasos apresurados, más aún en una organización que tiene como objetivo obtener el $100 \%$ de beneficio, esto hace que los trabajadores lleguen al cumplimiento estricto de metas, y en muchas ocasiones dejan de lado su valor humano, no solo perjudicando el desempeño laboral sino también la productividad de las actividades diarias del trabajador y a la empresa donde laboran. Hay que rescatar que al pasar los años se nota con más frecuencia las tantas tensiones laborales a las que se somete cada trabajador que laboran en diferentes empresas, logrando ser desencadenante y perjudicial en su desempeño laboral (Puma; Azaña, 2020). 
Por consiguiente, la expresión de ira manifiesta conductas agresivas; tal como muestran los datos del Observatorio Permanente de Riesgos Psicosociales de UGT, el $17 \%$ de los trabajadores han sido objeto de algún comportamiento violento en el lugar de trabajo, sin encontrar diferencias en lo que respecta al género. (Aragon, 2012). Entonces, diariamente estamos expuestos a agresiones físicas y verbales; estos problemas parten de una premisa y esta es la ira, nuestra sociedad está formada por personas iracundas que, en su mayoría, no logran reconocer expresar y aceptar adecuadamente emociones, como el saber controlar y expresar adecuadamente nuestras emociones; facilitando no solo nuestro estilo de vida, reduciendo el estrés y fortaleciendo la resiliencia, sino también nuestras relaciones interpersonales (Anélidos, 2008).

Entonces debido a las consecuencias que esto genera en los trabajadores, es necesario que las empresas no dejen a un lado a sus empleados ya que son el activo importante de la organización y de ellos depende el éxito de la empresa. Sabemos que no es posible dar un remedio específico para la prevención del estrés laboral, pero sin embargo es posible ofrecer orientación para prever el estrés en la organización. Como fundamento básico para una fuerza de trabajo con retos a su salud, los trabajadores o colaboradores tienen que estar motivados, sentirse seguros en su trabajo, satisfechos y percibir que tienen el control de su trabajo tanto en tiempo y espacio. La prevención del estrés laboral es una meta importante y se propone un proceso escalonado: compromiso de gerencia, crear la conciencia del estrés laboral en la organización, apoyo familiar, el uso de métodos iguales o estandarizados, retroalimentación y participación de cada empleado, y establecer metas con límites de tiempo. Beneficiando a familias, trabajadores de la unidad minera y la organización misma (Houtman, 2015).

A nivel teórico con respecto a la literatura científica, luego de una búsqueda en las principales bases de datos (Scielo, Redalyc, repositorio UNA - PUNO, UPeU, entre otros) no se hallaron resultados de investigación en la población de la región Puno, lo cual da notar que existe un desconocimiento científico respecto a estas variables. Es necesario generar evidencias empíricas.

Solorzano Ticona (2018) En el distrito de Santa
Lucia, provincia de Lampa, departamento de Puno - Perú, realizó una investigación titulada "Estrés laboral y su influencia en la ocurrencia de accidentes en la unidad minera Tacaza CIEMSA - PUNO 2017" teniendo como objetivo: el analizar la influencia del estrés laboral en la ocurrencia de accidentes en la unidad minera Tacaza - CIEMSA - Puno. La metodología aplicada fue el diseño de investigación descriptivo, inductivo por la aplicación de cuestionarios junto a ellos su descripción y desarrollo; la población de 189 trabajadores de la unidad minera Tacaza. Finalmente, el cuestionario utilizado como instrumento de encuesta se desarrolló mediante la revisión de las diversas publicaciones sobre el estrés laboral en los trabajadores. Los resultados muestran que se da la influencia del estrés laboral en la unidad minera de acuerdo con el análisis, la edad de más ocurrencia es de 30 a 37 años, la influencia del estrés laboral, en ocurrencia de accidentes por tiempo de servicio es entre 2 a 16 meses. Dando por conclusión que el estrés laboral es muy influyente en la ocurrencia de accidentes en la unidad minera.

En Arequipa, Arias (2012) realizó una investigación "Estrés laboral en trabajadores desde el enfoque de los sucesos vitales" cuyo objetivo es identificar los sucesos vitales más frecuentes que se relacionan con el estrés en los trabajadores de una empresa. Trabajó con una muestra de 100 trabajadores varones de una empresa dedicada a la asistencia y mantenimiento minero, con profesionales: ingenieros, administrativos y mecánicos de entre 19 a 55 años. Finalmente, el instrumento utilizado fue la escala de evaluación de reajuste social de Holmes y Rahe. el resultado indica cambios en los hábitos de alimentación, vida y sueño. Los administradores marcaron cambios de responsabilidades en el trabajo. Por otro lado, los ingenieros marcaron su cambio en su vida matrimonial sentimental y en días no laborables.

En Pimentel, Liza (2020) realizo la investigación "Estrés laboral y clima organizacional en los colaboradores de la empresa Buen Vivir S.A.C. 2020" La metodología fue de un diseño no experimental, de tipo descriptivo y correlacional, tomando 30 colaboradores. Por resultado se ve que el $100 \%$ de los colaboradores se encuentran en un nivel alto de estrés laboral y el $77 \%$ indican que tienen que trabajar con rapidez, asumir muchas tareas y ver que sea preciso el trabajo diario. 
Sin embargo en Lima, Medina (2018) en su investigación "expresión de ira, según su género, en estudiantes del quinto de secundaria de una institución educativa", la metodología es de tipo no probabilístico de estudio descriptivo comparativo, se tomó como muestra a 250 alumnos, el instrumento aplicado fue el inventario STAXI 2 que comprende la ira como estado y rasgo. Finalmente, como resultado no presentan diferencias, se afirma que las personas tanto femenino y masculino expresan su ira en similares niveles indicando que es debido al estilo de crianza similar entre ellos.

Por lo anteriormente expuesto, se tiene como objetivo determinar si existe relación significativa entre el estrés laboral y expresión de ira en trabajadores de una unidad minera en tiempos de covid - 19, Juliaca 2021. Los objetivos específicos procuran determinar la relación entre el estado de ira y estrés laboral; relación entre rasgo de ira y estrés laboral y finalmente, relación entre expresión - control de ira y estrés laboral.

\section{METODOLOGÍA}

En el presente estudio se optó por un diseño no experimental, puesto que el objetivo no era la modificación de la variable, sino el estudio típico del comportamiento de las variables en un ambiente natural; es de tipo correlación con la finalidad de estudiar la relación que existe entre las variables. El enfoque es cuantitativo por que el instrumento usado proporciona resultados numéricos las cuales serán procesados bajo esta índole y de corte transversal porque se aplicó una vez (Hernandez, Fernandez, \& Baptista, 2014)

\section{Participantes}

Respecto a la población considerada en el estudio, se contó con personas entre edades de 20 a 50 años, en la gran mayoría procedentes de la ciudad de Juliaca; estas personas pertenecen a una unidad minera en la provincia de San Román - Juliaca. Esta población alcanza los 60 sujetos. La muestra es de tipo no probabilístico ya que trabajamos en base bajo a criterios de inclusión y exclusión. Se tiene como meta encuestar a 60 participantes.

Los criterios de inclusión que se considera en el estudio son:
- Edad de 18 a 50 años en ambos sexos.

- Que accedan a participar del estudio.

- Estar registrado en la unidad minera.

Los criterios de exclusión que se considera en el estudio son:

- Ser menor de edad.

- No acceder a participar del estudio.

- No estar registrado en la unidad minera.

\section{Instrumentos}

\section{Estrés laboral}

Esta variable será medida a través del instrumento utilizado en la presente investigación, el cuestionario de estrés laboral publicado por la Organización Internacional del Trabajo OIT - Organización Mundial de la Salud OMS y de autoría por Ivancevich y Mattenson en 1989 y adaptado en el Perú por la Mg. Ángela Suárez Tunanñaña en el año 2013, su utilidad se encuentra en la detección del estrés laboral y la capacidad de predecir las fuentes de riesgos psicosociales, consta de 25 ítems, organizados en 7 áreas: clima organizacional, estructura organizacional, territorio organizacional, tecnología, influencia de líder, falta de cohesión y respaldo de grupo. La confiabilidad con el coeficiente alpha de Cronbach fue de 0.966 , lo que represente alta confiabilidad de la prueba (Suárez, 2013).

En la tabla 1 se observan los índices de acuerdo con la revisión del contenido en 4 jueces expertos, el índice alcanzado en el constructo total es de 0,98 este resultado indica que el instrumento es válido para su uso en investigación científica. En la dimensión clima organizacional tiene $V=0,938$, la dimensión estructura organizacional tiene $V=0,984$, en la dimensión territorio organizacional tiene $V=1$, la dimensión tecnología tiene $\mathrm{V}=1$, la dimensión influencia de líder tiene $V=1$, la dimensión falta de cohesión tiene $V=1$, sin embargo, en la dimensión respaldo de grupo tiene $V=1$ estos resultados indican que las dimensiones son válidas en cada uno de sus contenidos. 
Tabla 1

Validez de contenido para el instrumento estrés laboral de Ivancecivh y Mattenson 1989

\begin{tabular}{llllll} 
Dimensiones & Claridad & Congruencia & Contexto & Dom. del Constructo & V. de Aiken \\
Clima Organizacional & 0.75 & 1 & 1 & 1 & 0.938 \\
Estructura Org. & 0.938 & 1 & 1 & 1 & 0.984 \\
Territorio Org. & 1 & 1 & 1 & 1 & 1 \\
Tecnología & 1 & 1 & 1 & 1 & 1 \\
Influencia de líder & 1 & 1 & 1 & 1 & 1 \\
Fala de Cohesión & 1 & 1 & 1 & 1 & 1 \\
Respaldo del grupo & 1 & 1 & 1 & 1 & 1 \\
TOTAL & 0.955 & 1 & 1 & 1 & 0.989 \\
\hline
\end{tabular}

\section{Expresión de la ira}

El instrumento utilizado para medir la expresión a la ira en la presente investigación fue el inventario de expresión de ira Estado - Rasgo (STAXI 2), autoría por Charles D. Spielberger en 1999 y la adaptación peruana de la prueba fue llevada a cabo por la Br. en Psic. Sebastián Campos Mirella en el año 2016, su utilidad se encuentra en la evaluación de la respuesta emocional de ira. Consta de 49 ítems organizados en 3 escalas: estado, rasgo y expresión - control. Con 8 subescalas: deseos de expresar y sentimiento de enojo, temperamento iracundo y reacción de enojo y por último control externo e interno del enojo y expresión interna - externa del enojo; permitiendo obtener un índice de cada escala y subescala en un índice general de la prueba. En el nivel de confiabilidad, utilizaron el Alfa de Cronbach con un 95\% de nivel de confianza, en el que obtuvieron .917, .841 y .772 en la subescalas estado, rasgo y expresión, respectivamente (Mirella, 2016)

En la tabla 2 se observan los índices de acuerdo con la revisión del contenido en 4 jueces expertos, el índice alcanzado en el constructo total es de 1 , este resultado indica que el instrumento es válido para su uso en investigación científica. Respecto a la dimensión estado de ira tiene $V=1$, en la dimensión rasgo de ira tiene $V=1$, en la dimensión expresión de ira tiene $V=1$ estos resultados indican que este instrumento es válido en contenido.

Tabla 2

Validez de contenido para el instrumento expresión a la ira de Charles D. Spielberger 1999

\begin{tabular}{llllll} 
Dimensiones & Claridad & Congruencia & Contexto & D. Constructo & V. de Aiken \\
Estado de ira & 1 & 1 & 1 & 1 & 1 \\
Rasgo de ira & 1 & 1 & 1 & 1 & 1 \\
Expresión de ira & 1 & 1 & 1 & 1 & 1 \\
TOTAL & 1 & 1 & 1 & 1 & 1 \\
\hline
\end{tabular}

\section{Alfa De Cronbach}

En la tabla 3 observamos que los resultados de alfa de cronbach $(=, 961)$ nos muestra que es recomendable el uso del instrumento. 
Tabla 3

Confiabilidad de contenido para el instrumento estrés laboral de Ivancecivh y Mattenson 1989

\begin{tabular}{lll} 
Alfa de Cronbach & Alfa de Cronbach basada en elementos estandarizados & N de elementos \\
,961 &, 962 & 25 \\
\hline
\end{tabular}

En la tabla 4 observamos que los resultados de alfa de cronbach $(=, 943)$ nos muestra que es recomendable el uso del instrumento.

Tabla 4

Confiabilidad de contenido para el instrumento expresión a la ira de Charles D. Spielberger 1999

\begin{tabular}{lll} 
Alfa de Cronbach & Alfa de Cronbach basada en elementos estandarizados & N de elementos \\
\hline, 943 &, 955 & 49 \\
\hline
\end{tabular}

\section{Análisis de datos}

En la obtención de datos, se manejó la adaptación de Google Forms para aplicar cuestionarios virtuales, se compartió un enlace a la población objetiva, por medio del WhatsApp, encuestando a 60 trabajadores de la unidad minera, Juliaca. Para el análisis de los datos se utilizó Microsoft Office Excel, permitiendo recodificar puntuaciones cualitativas a cuantitativas, para luego pasar dichos datos convertidos al software SPSS en su versión 26. Se extrajo los estadísticos de frecuencia respecto a los datos sociodemográficos, de la misma manera se obtiene tablas de frecuencia de personas que se sitúan en determinados niveles por cada variable de estudio. Para determinar el tipo de distribución de las variables, se hará uso de la prueba de bondad de ajuste por Kolmegorov Smirnov, los resultados demuestran una distribución no normal, este último justifica el uso de la formula Rho de Spearman para determinar el índice de correlación entre las variables, de la misma manera, esta fórmula se utiliza, para identificar la relación entre las dimensiones y la variable.

\section{RESULTADOS}

\section{Resultados sociodemográficos}

En la tabla 5 se observa la población de estudio conformada por 60 trabajadores; en edad el $10.0 \%$ pertenece al rango de 20 a 21 años, el $68,3 \%$ pertenece al rango de 22 a 30 años, el $18,3 \%$ pertenece al rango de 31 a 39 años y el $3,3 \%$ al rango de 40 años a más. En sexo, el $90,0 \%$ a 54 varones y el $10,0 \%$ a 6 mujeres. En estado sentimental el $38,3 \%$ son 23 solteros, el $21,7 \%$ son 13 trabajadores con enamorado(a), el $8,3 \%$ son 5 comprometidos, el $1,7 \%$ es 1 casado, el $28,3 \%$ son 17 convivientes y el $1,7 \%$ es un 1 divorciado. En el grado, el 6,7\% representa a 4 participantes con secundaria completa, el $50,0 \%$ representa a 30 con el grado de técnico y el $43,3 \%$ representa a 26 participantes con universidad. Finalmente, en ocupación, el $16,7 \%$ son 10 electricistas, el $16,7 \%$ son 10 soldadores, el $48,3 \%$ son 29 mecánicos, el $8,3 \%$ son 5 ingenieros, el 3,3\% son 2 choferes y el $6,7 \%$ son 4 en el área de administración. 
Tabla 5

Análisis sociodemográficos de la población de estudio

\begin{tabular}{|c|c|c|}
\hline & $f$ & $\%$ \\
\hline \multicolumn{3}{|l|}{ Edad } \\
\hline 20 a 21 años & 6 & $10,0 \%$ \\
\hline 22 a 30 años & 41 & $68,3 \%$ \\
\hline 31 a 39 años & 11 & $18,3 \%$ \\
\hline 40 años a más & 2 & $3,3 \%$ \\
\hline \multicolumn{3}{|l|}{ Sexo } \\
\hline Varón & 54 & $90,0 \%$ \\
\hline Mujer & 6 & $10,0 \%$ \\
\hline \multicolumn{3}{|l|}{ Estado sentimental } \\
\hline Soltero & 23 & $38,3 \%$ \\
\hline Enamoramiento & 13 & $21,7 \%$ \\
\hline Comprometido & 5 & $8,3 \%$ \\
\hline Casado & 1 & $1,7 \%$ \\
\hline Conviviente & 17 & $28,3 \%$ \\
\hline Divorciado & 1 & $1,7 \%$ \\
\hline \multicolumn{3}{|l|}{ Grado } \\
\hline Secundaria Completa & 4 & $6,7 \%$ \\
\hline Técnico & 30 & $50,0 \%$ \\
\hline Universidad & 26 & $43,3 \%$ \\
\hline \multicolumn{3}{|l|}{ Ocupación } \\
\hline Electricista & 10 & $16,7 \%$ \\
\hline Soldador & 10 & $16,7 \%$ \\
\hline Mecánico & 29 & $48,3 \%$ \\
\hline Ingeniero & 5 & $8,3 \%$ \\
\hline Chofer & 2 & $3,3 \%$ \\
\hline Administración & 4 & $6,7 \%$ \\
\hline
\end{tabular}

\section{Análisis descriptivo}

En la tabla 6 se muestra los resultados del análisis descriptivo del nivel de estrés laboral en trabajadores en una unidad minera en la ciudad de Juliaca. Se encontró que el $81,7 \%$ de los trabajadores presentan un nivel bajo de estrés laboral, el $15,0 \%$ de los trabajadores presentan un nivel intermedio de estrés laboral y con un $3,3 \%$ de los trabajadores presentan un nivel de estrés laboral.

Tabla 6

Nivel de estrés laboral en los trabajadores de la unidad minera en tiempos de covid - 19, Juliaca 2021

\begin{tabular}{llllll} 
& & Frecuencia & Porcentaje & Porcentaje válido & Porcentaje acumulado \\
Válido & Bajo & 49 & 81,7 & 81,7 & 81,7 \\
& Intermedio & 9 & 15,0 & 15,0 & 96,7 \\
& Estrés & 2 & 3,3 & 3,3 & 100,0 \\
& Total & 60 & 100,0 & 100,0 & \\
\hline
\end{tabular}


En la tabla 7 se muestran los resultados de los análisis descriptivos del nivel de expresión de ira en trabajadores en una unidad minera en la ciudad de Juliaca 2021. Respecto al nivel de expresión de ira se observa un alto nivel de expresión de ira con un 25,0\% que corresponde a 15 participantes, en el nivel moderado de expresión de ira se evidencia un $45,0 \%$ que corresponde a 27 participantes y finalmente en el nivel bajo de expresión de ira se muestra un $30,0 \%$ que corresponde a 18 participantes.

Tabla 7

Nivel de expresión de ira en los trabajadores de una unidad minera en tiempos de covid - 19, Juliaca 2021

\begin{tabular}{llllll} 
& & Frecuencia & Porcentaje & Porcentaje válido & Porcentaje acumulado \\
Válido & Bajo & 18 & 30,0 & 30,0 & 30,0 \\
& Moderado & 27 & 45,0 & 45,0 & 75,0 \\
& Alto & 15 & 25,0 & 25,0 & 100,0 \\
& Total & 60 & 100,0 & 100,0 & \\
\hline
\end{tabular}

\section{Prueba de normalidad}

En la tabla 8, se observa las pruebas de normalidad para la variable estrés laboral con Sig. ,027 y en la variable expresión de ira con Sig. ,200. Por lo tanto, las variables tienen una distribución no normal teniendo un $\mathrm{p}<0.05$ en una de las variables, por lo cual se trabajará con estadísticos no paramétrico.

Tabla 8

Análisis de normalidad mediante la prueba de Kolmogorov - Smirnov para una población

\begin{tabular}{|c|c|c|c|}
\hline & \multicolumn{3}{|c|}{ Kolmogorov-Smirnova } \\
\hline & Estadístico & $\mathrm{gl}$ & Sig. \\
\hline Estrés Laboral & ,122 & 60 & ,027 \\
\hline Expresión de Ira & ,086 & 60 & ,200* \\
\hline
\end{tabular}

*. Esto es un límite inferior de la significación verdadera.

a. Corrección de significación de Lilliefors

\section{Correlación de las variables}

En la tabla 9 se encontró correlación entre las variables estrés laboral y expresión de ira con $(r h o=, 577)$ siendo de intensidad media, y un nivel de Sig. $=, 000$ cuyo valor es menor al 0,05 entonces podemos afirmar que si existe relación estadísticamente significativa entre estrés laboral y expresión de ira.

Tabla 9

Análisis de correlación Rho de Spearman de las variables estrés laboral y expresión de ira

\begin{tabular}{lllll} 
Rho de Spearman & \multirow{2}{*}{ Estrés Laboral } & Coeficiente de correlación & 1,000 &, $577^{* *}$ \\
& & Sig. (bilateral) & &, 000 \\
& & $\mathrm{~N}$ & 60 & 60 \\
& \multirow{2}{*}{ Expresión Ira } & Coeficiente de correlación &, $577^{* *}$ & 1,000 \\
& Sig. (bilateral) &, 000 & \\
& $\mathrm{~N}$ & 60 & 60 \\
\hline
\end{tabular}

**. La correlación es significativa en el nivel 0,01 (bilateral). 
En la tabla 10 se observan las correlaciones entre la variable estrés laboral y la dimensión expresión de ira, cuyo resultado evidencia correlación directa $(\mathrm{Rho}=, 499$; Sig. $<0,05)$, para la correlación entre la variable estrés laboral y la dimensión rasgo existe una relación significativa con valor $(\mathrm{Rho}=, 575$; Sig. $<0,05)$ y finalmente entre la variable estrés laboral y la dimensión expresión - control de ira se evidencia $(\mathrm{Rho}=, 391$; Sig. $<0,05)$ mostrando relación significativa entre la variable estrés laboral y las dimensiones de la variable expresión de ira.

Tabla 10

Correlación de la variable estrés laboral y las dimensiones de la variable expresión de ira

\begin{tabular}{|c|c|c|c|c|c|c|}
\hline & & & $\begin{array}{l}\text { Estrés } \\
\text { Laboral }\end{array}$ & Estado & Rasgo & $\begin{array}{l}\text { Expresión } \\
\text { y Control }\end{array}$ \\
\hline \multirow[t]{3}{*}{ Rho de Spearman } & \multirow{3}{*}{$\begin{array}{l}\text { Estrés La- } \\
\text { boral }\end{array}$} & Coeficiente de correlación & 1,000 &, $499^{* *}$ &, $575^{* *}$ &, $391^{\star *}$ \\
\hline & & Sig. (bilateral) & & ,000 &, 000 &, 002 \\
\hline & & $\mathrm{N}$ & 60 & 60 & 60 & 60 \\
\hline
\end{tabular}

**. La correlación es significativa en el nivel 0,01 (bilateral).

*. La correlación es significativa en el nivel 0,05 (bilateral).

\section{DISCUSIÓN}

El objetivo general del estudio fue determinar la relación entre estrés laboral y la expresión de ira en los trabajadores de una unidad minera en tiempos de covid - 19 en la ciudad de Juliaca 2021. A partir del procedimiento estadístico los resultados indican que existe relación directa a nivel medio (rho=,577) y estadísticamente significativa $(p<, 05)$ indicando que existe relación directa y significativa entre estrés laboral y expresión de ira. Con lo encontrado en la investigación de Trinidad \& Llontop (2020) menciona en su investigación "estrés laboral y relación con el desempeño laboral de los trabajadores de la empresa DSE Ingeniería SAC - Lima 2020" una relación significativa utilizando un diseño no experimental de corte transversal; en uno de sus principales hallazgos demostró que si existe una relación significativa entre la variable estrés laboral y la dimensión comportamiento de los trabajadores con Tau-b de kendall ( $\mathrm{t}=0.688)$, con un nivel de significancia menor al $5 \%$ (sig.<0.05); esto implica que en los trabajadores de la empresa muestren crecientes niveles de estrés laboral y su nivel de expresión de ira tiende a ser incrementada. Estos resultados guardan relación con Molina (2020) quien realizó la investigación "estrés laboral en personal administrativo de una institución de formación policial en tiempos de covid - 19 de lima, 2020" sus resultados indicaron que acepta su ha y desaprueba la hn presentando un valor $<0,05$, obteniendo un $95 \%$ de nivel alto de estrés laboral en el trabajador, paralelamente existen conflictos como la discordia generalmente en el ambiente laboral, desarrollado en cada colaborador por lo que piensa y lo que considera, percibiendo que existen estos conflictos en varias empresas; lo toman como bueno y malo dependiendo de la perspectiva, por ejemplo, los conflictos buenos en varias oportunidades resultan de manera beneficiosa para la empresa pero por otro lado aquellos conflictos malos obstaculizan actividades de la organización.

Con respecto al objetivo específico entre la dimensión estado de ira y la variable estrés laboral $($ rho $=, 499)$ existe relación significativa, ya que poseen correlación positiva, con una intensidad media, esta asociación viene a ser estadísticamente significativa $(p=, 000)$. De acuerdo con (Schiffman, Lazar y Wisenblit (2014) así como se citó en Liza Neciosup (2020) el estrés laboral es concepto de una secuencia de resultados psico - fisio y conductual; propio de impacto continuo de alguno o más divisores estresantes entre mismos trabajadores de una compañía. En su investigación "estrés laboral y clima organizacional en colaboradores de la empresa buen vivir S.A.C. 2020" encontró que los colaboradores en general trabajan con rapidez, recibiendo arduo trabajo diariamente y asumen muchos compromisos injustamente; todo esto producto del nivel alto de estrés laboral.

De igual forma existe relación significativa entre la dimensión rasgo y la variable estrés laboral, con una correlación positiva $(\mathrm{rho}=, 575)$ 
de intensidad media, esta asociación viene a ser estadísticamente significativa $(p=, 002)$; por consiguiente se ve que otro resultado a causa del estrés laboral es el rasgo de ira coincidiendo con la investigación de Graneros (2018) quien indica que el $80 \%$ del personal mantiene un alto nivel de estrés laboral causados por los horarios rotativos y diferencias entre ellos; agregando que existe como resultado las dificultades en las relaciones sociales y familiares, al mismo tiempo resulta efecto negativo en la pareja o conyugue y en la crianza de los hijos por falta del horario de reposo en común dificultando la programación familiar.

En cuanto a la relación entre la dimensión expresión - control de ira y la variable estrés laboral con una correlación positiva $($ rho $=, 391)$ de intensidad baja, esta asociación viene a ser estadísticamente significativa $(p=, 000)$. Por eso González Acabal (2014) en su investigación "estrés y desempeño laboral" la eficacia laboral da como producto a la empresa que su empleado labore con satisfacción, asumiendo que el rendimiento será dependiendo de su comportamiento de acuerdo al clima organizacional, esto genera que el empleado tiene la obligación de cumplir con metas mensuales establecidas, generando estrés cuando no logran cumplir las metas, y tanto como su puesto de trabajo y su salario se ven afectados generando rivalidad entre sí mismos y accidentes dentro de la empresa como nos indica Solorzano (2018) en su investigación "estrés laboral y su influencia en la ocurrencia de accidentes en la unidad minera tacaza - ciemsa - Puno 2017" menciona que el estrés laboral si influye en el acontecimiento de accidentes, observando el valor que se tiene en cuanto al trabajo desempeñado, además se ve en lo encontrado, que el estrés laboral no es algo trivial porque causa la alteración en la conducta del empleado, agraviando la salud y condición de vida, esto se nota gracias a síntomas "organizativos" rotación del personal, bajo rendimiento, el alto ausentismo, desánimo, falta de innovación y la baja productividad. Sierra, Ortega y Zubeidat (2003) en su investigación "ansiedad, angustia y estrés" el estrés viene siendo un estímulo como respuesta e interacción entre colaboradores creando una amenaza para sus valores morales y a su integridad física psicológica.

A medida que las personas controlen sus niveles de estrés laboral, sus niveles de expresión de ira reducirán. Finalmente, este estudio tuvo limitaciones a causa de no contar con el tiempo suficiente, estudios muy escasos enfocados a trabajadores en la minería y no contar con más unidades mineras; se recomienda que los próximos estudios añadan otras variables.

\section{Correspondencia}

\section{Luiggi J.Zamora Deza}

Correo electrónico:

luiggi.zd@upeu.edu.pe

\section{REFERENCIAS}

Arias Gallegos, W. L. (2012). occupational stress of workers analyzed from an approach to vital events. Revista Cubana de Salud Publica, 38(4), 525-535. https://doi.org/10.1590/S086434662012000400004

Aragon. (2012). https://www.aragon.es/ documents/20127/674325/violecia_ambito_ laboral.pdf/a619572a-3a40-f681-aeecc6fd989182e7

Aragon. (2012). Obtenido de https://www.aragon. es/documents/20127/674325/violecia_ambito_ laboral.pdf/a619572a-3a40-f681-aeecc6fd989182e7

Becerra Hernandez, R. M. (2020). Estrés laboral y calidad de vida en tiempos de pandemia en docentes de escuelas con quintil 1 - cajamarca. Persepsi masyarakat terhadap perawatan ortodontik yang dilakukan oleh pihak non profesional, 53(9), 1689-1699.

Diario Correo. (2021). Diario correo. https:// diariocorreo.pe/economia/estres-laboralcomo-afecta-y-que-hacer-para-manejarloproductividad-laboral-trabajo-remoto-exceso-detrabajo-trabajandocom-visma-latinoamerica-relaciones-interpersonales-pandemia-desempleocrisis-ncze-noticia/?Ref=dcr

Diario Correo. (2021). Diario Correo. https:// diariocorreo.pe/economia/estres-laboralcomo-afecta-y-que-hacer-para-manejarloproductividad-laboral-trabajo-remoto-exceso-detrabajo-trabajandocom-visma-latinoamerica-relaciones-interpersonales-pandemia-desempleocrisis-ncze-noticia/?ref=dcr 
Expok. (2017). Comunicación de sustentabilidad y RSE. https://www.expoknews.com/quienessufren-mayor-estres-laboral-en-el-mundo/

Graneros, B. (2018). Estrés laboral en personal policial con turnos rotativos y fijos. 73. Http:// imgbiblio.vaneduc.edu.ar/fulltext/files/TC127260. pdf

Gan Bustos, F., \& Berbel Giménez , G. (2011). Manual de recursos humanos. Barcelona: UOC. Obtenido de http://reader.digitalbooks.pro/book/ preview/28329/chap0.xhtml/-?1624076775229

González Acabal, M. R. Mayo (2014). Estres y desempeño laboral. https://www. academia.edu/28225252/_estr\%c3\%89s_y desempe\%c3\%910_laboral_estudio_realizado_ en_serviteca_altense_de_quetzaltenango_tesis

Houtman, I., Jettinghoff, K., \& Cedillo, L. (2015). Sensibilizando sobre el estrés laboral en los países en desarrollo. Un riesgo moderno en un ambiente de trabajo tradicional. Consejos para empleadores y representantes de los trabajadores. World Health Organization, 6, 1-41. https://apps.who.int/iris/bitstream/ handle/10665/43770/9789243591650_spa.pdf;js essionid=2b432e7d1aec4480b6c2526151b 8f3fb? sequence $=1$

Liza Neciosup, J. J. (2020). Estrés laboral y clima organizacional en los colaboradores de la empresa buen vivir s.a.c. 2020. 122.

Medina Rios, P. V. (2018). universidad ricardo palma facultad de ciencias biológicas. september.

Molina Ormeño, L. M. (2020). Facultad de ciencias de la salud. Transtornos Alimenticios, $41 . \quad$ http://repositorio.uwiener.edu.pe/ handle/123456789/2216\%0Ahttp://www.scielo. br/pdf/ean/v13n2/v13n2a08.pdf. 2009 abr-jun; 13(2).

Mendoza, S. (2020). Visma Blog. Obtenido de https:// latam.visma.com/blog/estres-laboral-cronicouna-espiral-silenciosa/

Mirella, S. C. (2016). Propiedades psicométricas del inventario de expresión ira estado - rasgo en los alumnos de $5^{\circ}$ de secundaria del distrito de paiján.

OIT. (2003). Organización internacional del trabajo. https://www.ilo.org/sector/activities/topics/ violence-and-stress/lang--es/index.htm

OIT. (2003). Organización internacional del trabajo. https://www.ilo.org/sector/activities/topics/ violence-and-stress/lang--es/index.htm
Puma Chambi, Noemi; Azaña Laura, V. (2020). Universidad peruana union. Dirección General de Investigación, 1-93. https:// drive.google.com/file/d/1_IR8G2xOKhcG2YVMyc1XXEeJ99GQQ2s/view

Sierra, J., Ortega, V., \& Zubeidat, I. (2003). Ansiedad, angustia y estrés: tres conceptos. Revista MalEstar e Subjetividade, 3(1), 10-59. https:// periodicos.unifor.br/rmes/article/view/1159

Solorzano Ticona, Y. W. (2018). Estres laboral y su influencia en la ocurrencia de accidentes en la unidad minera Tacaza - Ciemza Puno 2017. Tesis una -puno, 17. https:// alicia.concytec.gob.pe/vufind/record/ rnap_9a9a7ecd586e30ffc1990aae18533ee3

Suárez, A. (2013). Adaptación de la escala de estrés laboral de la oit-oms en trabajadores de 25 a 35 años de edad de un contact center de lima. revista psiquemag, 2, 33-50. http://blog.ucvlima. edu.pe/index.php/psiquemag/article/view/8/8

Sampieri, R. H., Collado, C. F., \& Lucio, P. B. (2014). Metodologia de la Investigación. Mexico: mcgrawhill / interamericana editores, s.a. de c.v.

Trinidad, F., \& Llontop, M. (2020). Escuela de Posgrado biometría. Psikologi Perkembangan, October 2019, 1-126. http://repositorio.uncp.edu. pe/bitstream/handle/UNCP/3000/Silva Acosta. pdf? sequence $=1$ \&isAllowed $=y \% 0 A$ https: $/ /$ repositorio.comillas.edu/xmlui/ handle/11531/1046

Tello, L. (2021). El 73\% de trabajadores indica que carga laboral se incrementó entre 1 y 5 horas al día. Gestion, págs. https://gestion.pe/economia/ management-empleo/el-73-de-trabajadoresindica-que-carga-laboral-se-incremento-entre-1y-5-horas-al-dia-noticia/?ref=gesr.

Recibido: 21/06/2021

Aceptado: 15/09/2021 